\title{
A Hierarchical Registration Algorithm based on Comparative Centerline Assessment Technique for Evaluating Altered Tortuosity in Cerebral Vessels
}

\author{
Abanti Shama Afroz \\ Lecturer, AIUB \\ 41/C, Road 4A \\ Dhanmondi, Dhaka
}

\author{
Puspita Saha \\ Student, AIUB \\ Shojon Tower 2 \\ 3 Shegunbagicha, Dhaka
}

\begin{abstract}
Three-dimensional cerebral registration is mostly performed with atlas based methods. However, in case of compact and pre-constructed (segmented) regions of interest (ROIs) involving only blood vessels which are prone to torturous changes; atlas-based approach does not offer the best output. This article suggests a hierarchical (top to bottom) skeleton based registration approach for similar cases. The method has been applied on five sets of cerebral artery locations with aneurysms in order to evaluate their post invasive structural changes. The algorithm works in a semi-automatic manner where the bifurcation zone has been selected as the reference zone. This landmark matching approach works as the basis of the initial stage, coarse affine transformation. The non-rigid intermediate stage is optional and is dependent on the difference of the comparative angular orientation of the models in three dimensional space. Afterward, a third stage of iterative affine transformation is applied for finer adjustments if there is scope for any. Once registered with limiting boundaries, the branch by branch structural comparisons are interpreted quantitatively with box and whisker plots. In order to verify the proposed method, overlapping for one of the fifteen branch sets has also been evaluated with dice similarity indices. The resulting comparison gives a good support in favour of the proposed method.
\end{abstract}

\section{General Terms}

Medical image registration, 3D volume registration, skeleton based registration.

\section{Keywords}

Pre-segmented neural blood vessels, centerline matching, auto landmark identification

\section{INTRODUCTION}

Physiological system modeling is a significant branch of study in the biomedical engineering field where detailed 3 dimensional solid structures of organs with pathological changes are generated, processed and compared for evaluating patients' conditions. These models are extracted from 3 dimensional volume images like magnetic resonance imaging (MRI) or computed tomography (CT) scans.

Advanced and specialized image processing techniques are utilized for these segmentation (generation) and post processing of patient specific organ models [1], [2]. Multiple software programs have been emerged for performing such operations.
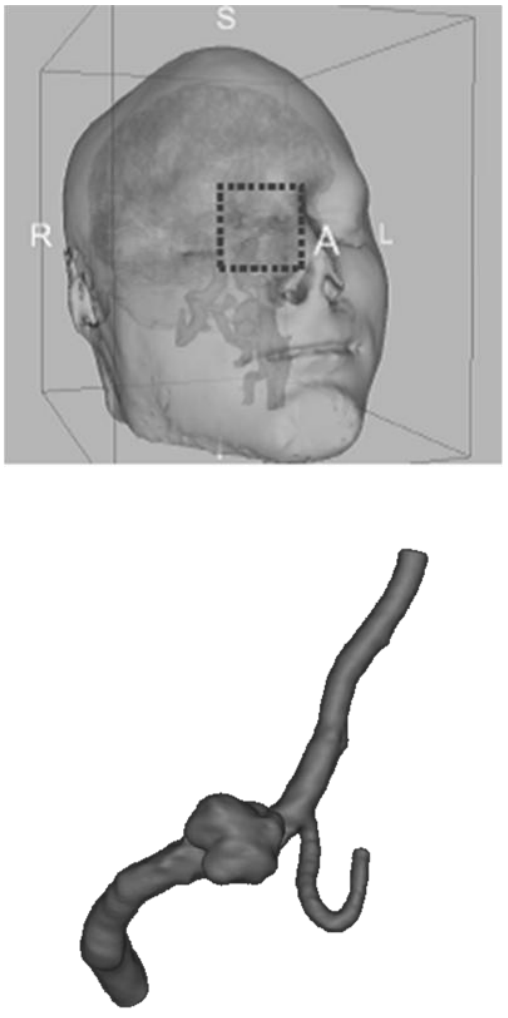

Fig 1: Segmentation of diseased blood vessel

However, being a developing research field, there is an issue of cross software platform matching when the intermediate stage data need to be processed with multiple programs. This particular study focuses on a similar specialized scenario where 2 sets of pre segmented cerebral blood vessels are available in their pre operative and post operative conditions [3]. In order to analyse the surgery performance, the pre and post operative blood vessels need to be compared. This only becomes more relevant when they are properly aligned and superimposed on one another.

Medical image registration at multiple stages itself is a vast field of study. Relevant literature review indicates that in case of many tree-like topologies as in pulmonary network or retinal microvasculature network, skeleton based registration gives better output [4], [5]. While cerebral registrations are mostly done with the atlas-based method, many a times with non rigid registration, owing to the fact that the cerebral zone 
holds so many components [6-10]. One feature of this atlasbased approach is that the registration tasks are mostly done with data from intermediate stages of segmentation i.e., $3 \mathrm{~d}$ model generation from volume images. So, the initial stage (raw) data, corresponding label maps (intermediate stage) and segmented models (final stage) - all have to be available at the same time for a certain registration task. As a consequence, in case of studies involving cross software platforms, matching of data formats becomes a restrictive issue.

This study is based on 5 sets of data which are available in their raw (DICOM data) and final stage (.stl surface models) versions. Also, the patient data were collected at their operative ROIs where only the aneurysm and neighbouring blood vessels were visible. Hence, the background was not optimal for application of atlas-based registration due to the presence of inadequate number of landmarks. Therefore, a hierarchical, semi-automated and skeleton-based alternative approach was outlined which can perform registration of the end stage $3 \mathrm{~d}$ models for evaluating surgery performance of cerebral blood vessel intervention.

\section{MATERIALS \& METHODS}

\subsection{Data}

Using manual approach 5 sets of diseased blood vessels have been segmented at the region of each set's interest (ROI) which holds a cerebral aneurysm i.e., pathological dilation of arteries in author's previous work. All the arteries had aneurismal conditions and were treated with stent supported coiling. Each set consists of two models, one in its preoperative stage and the other in post-operative stage. The 10 vessel models that have been used in this study were generated during author's Master's thesis[3].

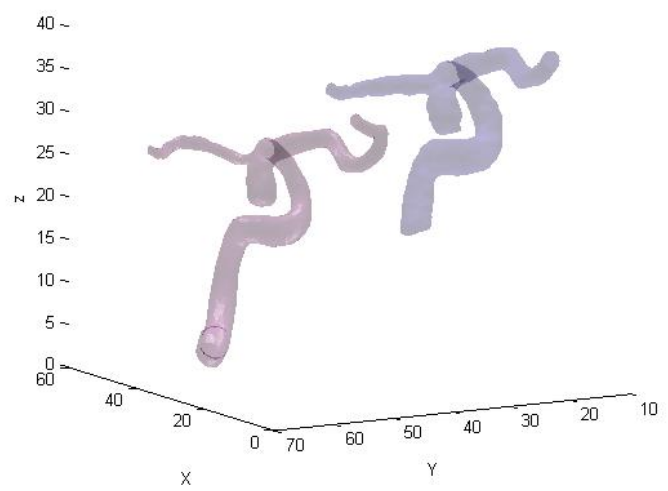

Fig 2: Segmented arteries for registration

\subsection{Algorithm}

The process of registering a pair of three dimensional surface volume followed a 'coarse to fine' transformation sequence with an intermediate and optional non-rigid shear transformation stage.

The algorithm can be summarized as:

- Centerline extraction

- Bifurcation based land landmark identification

- Coarse, affine transformation.

- Intermediate, non-rigid shear transformation.

- Fine iterative transformation

\subsection{Centerline Extraction}

The VMTK software program was used to extract the centerlines. This part of the task needs human interaction. The objective of using this toolkit was to extract accurate centerlines of bifurcated artery structures with the minimum user interface. Only the inlet and outlet surfaces were cropped and indicated by the user. The toolkit uses a topological analysis algorithm which splits the bifurcating structures in four zones, numbered as 0,1, 2 and 3 [11]. An example of the marked groups can be seen in figure 5. the steps of extracting the centerline can be listed as below -

- At first, clipping followed by capping was performed in the surface planes which were later considered as blood inlet and outlets.

- Secondly, the starting (inlet) surface and ending (outlet) surfaces were explicitly indicated.

- The inherent logic of the toolkit automatically calculated the centerlines and divided into 4 distinctive sections: inlet (0), bifurcating zone (1), first outlet (2) and second outlet (3). This grouping was maintained for the rest of this study.

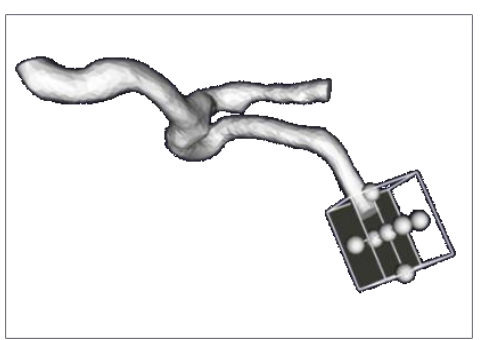

Fig 3: Clipping and capping of end surfaces

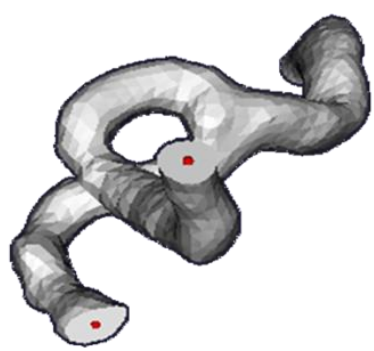

Fig 4: Manually assigning inlet and outlets

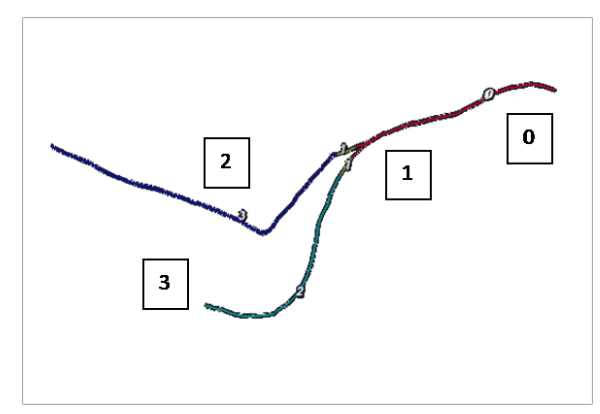

Fig 5: Grouping of tree branch like topology

\subsection{Landmark Identification}

All the vessel structures were similar in the sense that the aneurysms were at bifurcating zone. The VMTK organized the centrelines in such a way that 'zone 0' and 'zone 1' hold points for both the branches. Considering this pattern, the first point of the 'zone 1' or the bifurcating zone was treated as the 
bifurcation landmark. The coarse transformation was based on this comparative landmarks positions.

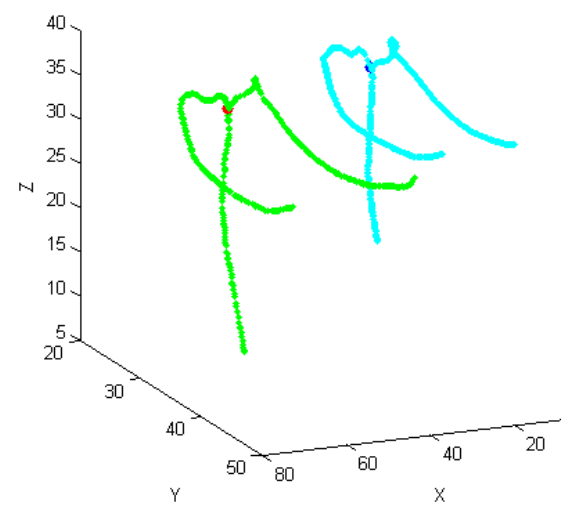

Fig 6: bifurcation landmark identification in MATLAB

\subsection{Coarse Affine Transformation}

The following equations were used for primary level coarse registration -

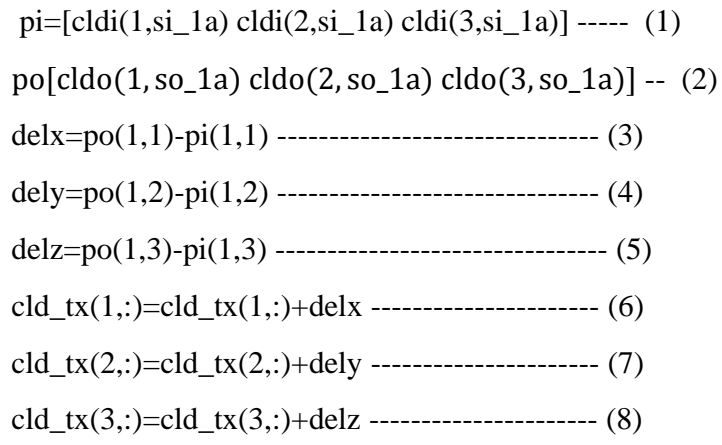

cld_tx $(1,:)=$ cld_tx $(1,:)+$ delx

cld_tx $(2,:)=$ cld_tx $(2,:)+$ dely

cld_tx $(3,:)=$ cld_tx $(3,:)+$ delz

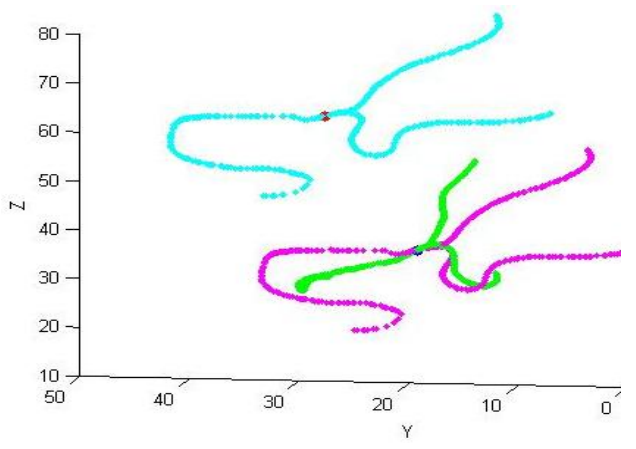

Fig 7: Coarse affine transformation

\subsection{Intermediate Shear Transformation}

This stage will be applicable only when 3 dimensional rotations are needed. This code fragment rotates the structure in 2 dimensional space and the equation is as followed -

$\operatorname{cld\_ shear}(\mathrm{q}, \mathrm{i})=\operatorname{cld}(\mathrm{q}, \mathrm{i})-\mathrm{k} \_$shear* $\left(\operatorname{cld}\left(\mathrm{p}, \mathrm{n} \_\mathrm{pivot}\right)-\operatorname{cld}(\mathrm{p}, \mathrm{i})\right) ;(9)$

At this stage, it is important to properly adjust the pivotal point aka the center of rotation and the shear factor values in 2 dimensional space

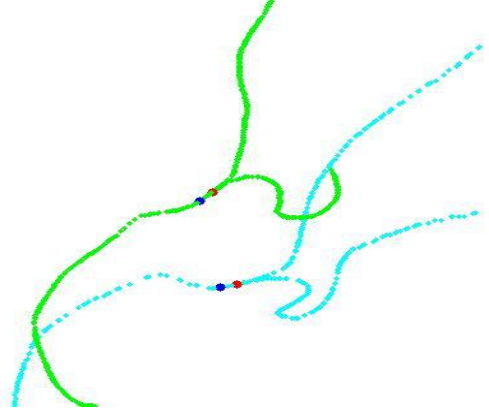

Fig 8: Non-rigid transformation on centre-lines

\subsection{Fine Iterative Registration}

Since the basic landmark is dependent on bifurcation geometry, the fine iterative operation is performed again in three dimensional domain, in total in nine steps. After performing each iterative step the performance analysis operation is calculated by determining one of 10 possibilities that has the best distance matrix having lower values of mean, maximum and minimum distances.

\section{PERFORMANCE ANALYSIS}

\subsection{Boundary Identification}

In order to evaluate the performance of the proposed multi stage registration, all the three equivalent extremities were identified. The steps are as followed -

- Sharp angular changes (if any) in each of the three branches were initially identified. In the presence of such changes (less than 5 degrees of changes in total for three axes) the curving points (one or multiple) were identified. The later part required user's manual interaction for identifying the corresponding limiting boundary between pre and post operative cases.

- In case of arteries without sharp turns, a code was used to identify the shortest of the two corresponding branches and its equivalent length from the other branch was considered to be the limiting boundary.

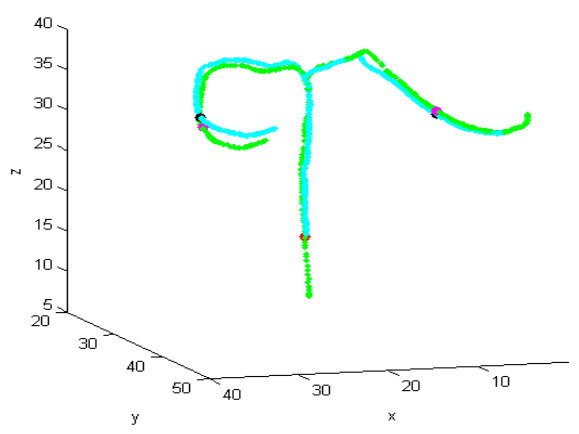

Fig 9: Boundary identification and reference line

\subsection{Reference for Branch Overlap}

For each of the three branches in every set (' 0 ', ' 2 ' and ' 3 '), the post operative branches starting and boundary end points taken were for reference and used to construct a straight line. Then, two distance matrices of all the points from both the branches (pre and post operative cases) were generated which hold the distances of the points from the reference line. These matrices were used together to measure the liner overlap of the 3 dimensional centrelines. The reference line for branch 3 
has been indicated in figure 9 with black dots. All the corresponding branch-points' vertical distances from this line are to be measured for generating the distance matrix. The hierarchical registration outputs can later be plotted for observing the registration outcome. Such a registration outcome for one of the 15 branches is plotted in figure 10 .

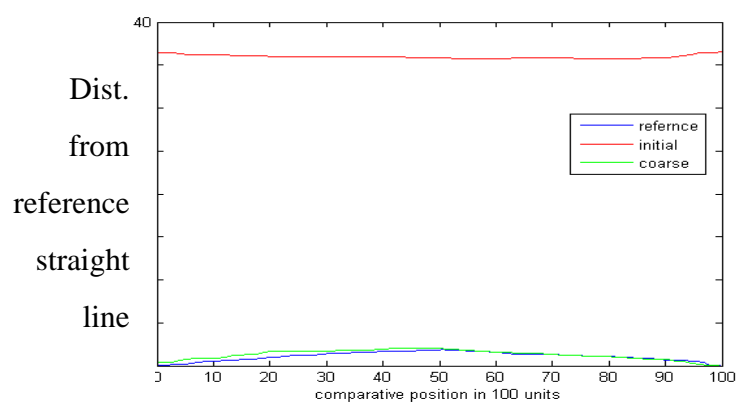

Fig 10: Plot of distance matrix for different transformation stages.

Comparative position in 100 units

\section{RESULTS}

\subsection{Superposition of Models}

- Figure 11-15 depict that the proposed registration approach successfully superimposed 5 sets of centerlines depending on the bifurcation based landmark approach.

- Once the centerlines (or corresponding surfaced models) are registered in this manner, evaluating vessel straightening or narrowing effect becomes computationally more efficient.

- Only one of the 5 sets required the intermediate non-rigid registration stage while other sets provide good results with the rigid affine transformation method.

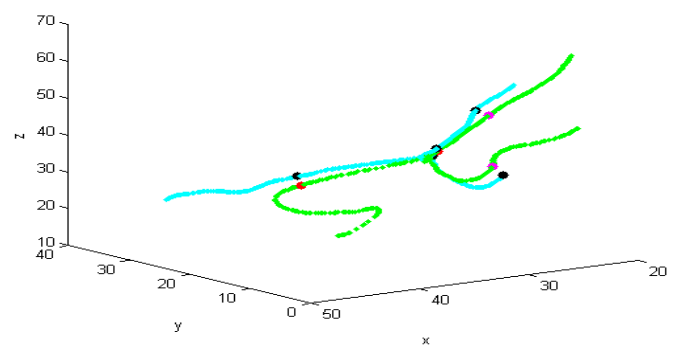

Fig 11: Registered output of set 1

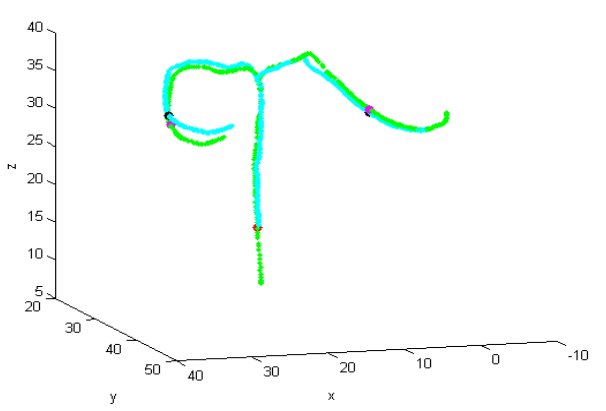

Fig 12: Registered output of set 2

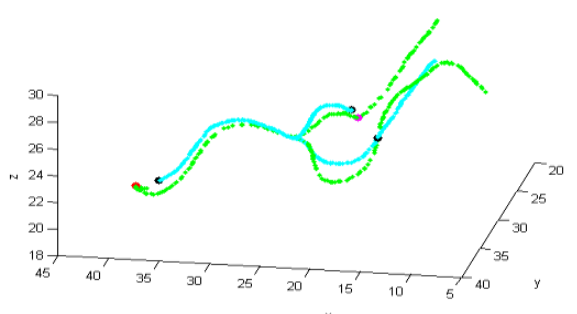

Fig 13: Registered output of set 3

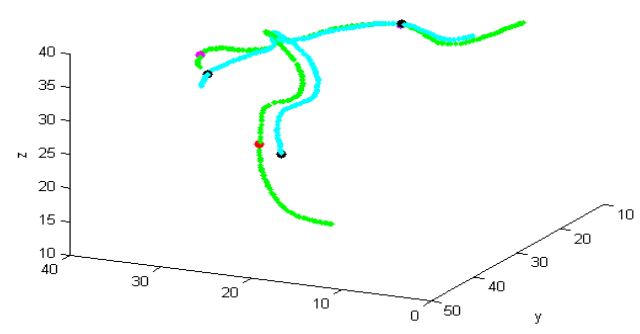

Fig 14: Registered output of set 4

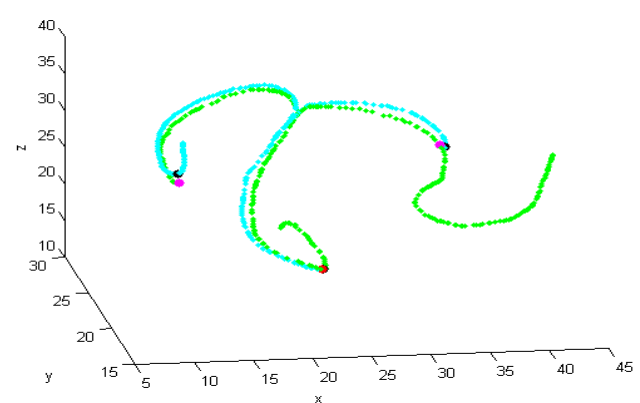

Fig 15: Registered output of set 5

\subsection{Quantification of torturous changes}

In order to evaluate structural changes between pre and post operative conditions, we need to consider each sets' both the whisker plots distribution which indicate maximum, $3^{\text {rd }}$ quartile, $2^{\text {nd }}$ quartile and minimum distribution values. The set with small differences in maximum and quartile distributions indicate minor changes while greater differences indicate large displacements. Therefore, by assessing table 1 and corresponding plot in figure 16 it can be inferred that -

- Post surgery spatial changes can be as high as about $7 \mathrm{~mm}$, as can be seen for set 1 .

- Only one of the five sets (set 2) indicate minor differences, 2 sets (set 1 and 3 ) are found to have large spatial changes (change in maximum values) while the rest 2 (set 4 and 5) indicate significant differences in terms of quartile distribution.

- All these observations can be summarized as stent supported coil deployment of aneurysms provide significant structural changes in aneurysms. It can be noted that similar observations were also recorded by [12], [13].

The set with small differences in maximum and quartile distributions indicate minor changes while greater differences indicate large displacements. 


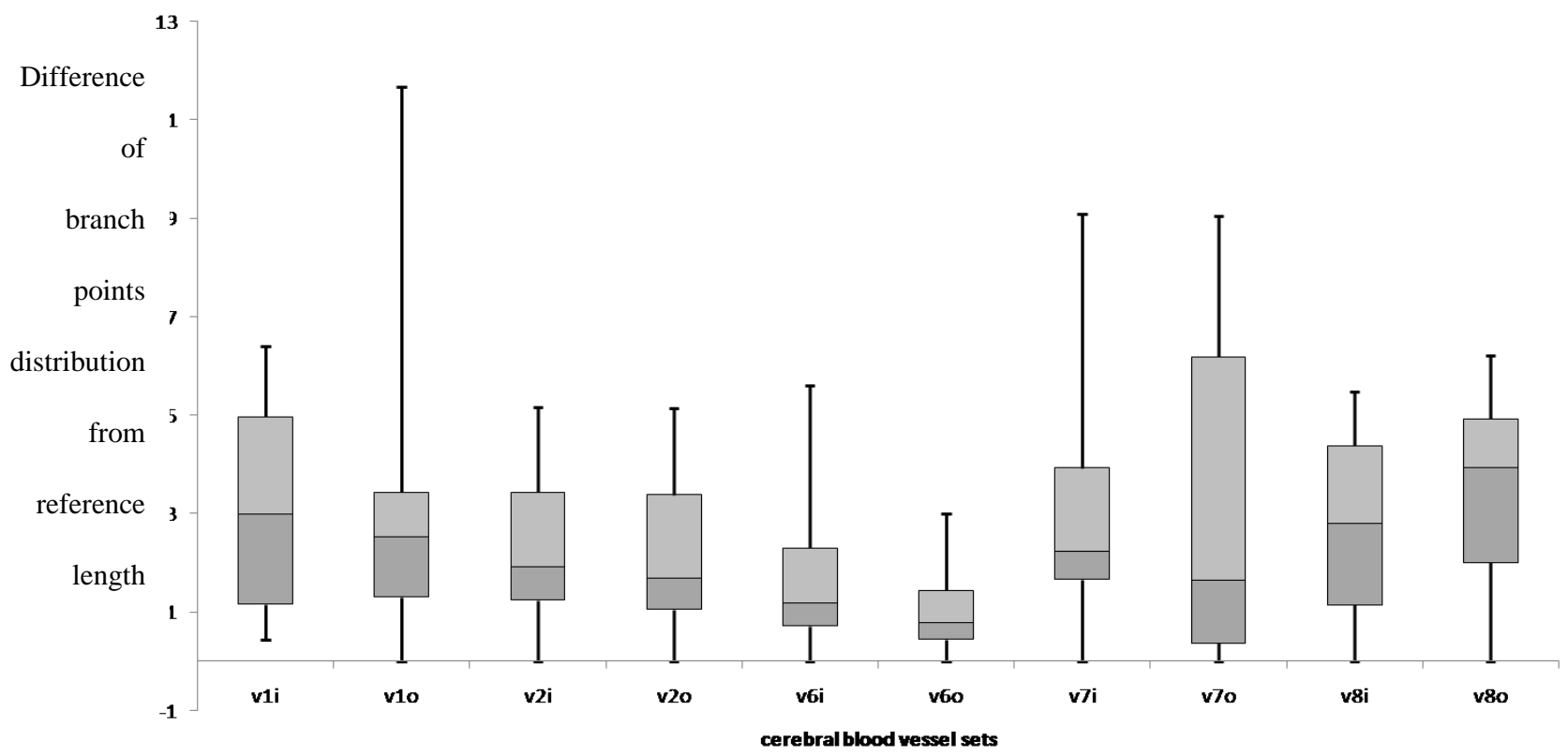

Cerebral blood vessel sets $(1,2,3,4 \& 5)$ in pre-operative (i) and post-operative(o) stages

Fig 16: Whisker plot distribution of distance matrices

Table 1. Comparison of overlapped branches

\begin{tabular}{|c|c|c|c|c|c|}
\hline & Min & q1 & $\mathrm{q} 2$ & $\mathrm{q} 3$ & $\max$ \\
\hline v1i & 0.4455 & $\begin{array}{c}0.712 \\
003\end{array}$ & $\begin{array}{c}1.8403 \\
6\end{array}$ & $\begin{array}{c}1.9652 \\
86\end{array}$ & 1.44081 \\
\hline v1o & 0 & $\begin{array}{c}1.304 \\
164\end{array}$ & $\begin{array}{c}1.2226 \\
57\end{array}$ & $\begin{array}{c}0.9066 \\
72\end{array}$ & $\begin{array}{c}8.23263 \\
2\end{array}$ \\
\hline $\mathrm{v} 2 \mathrm{i}$ & 0 & $\begin{array}{c}1.239 \\
219\end{array}$ & $\begin{array}{c}0.6749 \\
3\end{array}$ & $\begin{array}{c}1.5174 \\
98\end{array}$ & $\begin{array}{c}1.73744 \\
9\end{array}$ \\
\hline v2o & 0 & $\begin{array}{c}1.053 \\
503\end{array}$ & $\begin{array}{c}0.6411 \\
68\end{array}$ & $\begin{array}{c}1.6833 \\
29\end{array}$ & $\begin{array}{c}1.76199 \\
6\end{array}$ \\
\hline v6i & $\begin{array}{c}3.5293 \\
8 \mathrm{E}-15\end{array}$ & $\begin{array}{c}0.716 \\
463\end{array}$ & $\begin{array}{c}0.4595 \\
87\end{array}$ & $\begin{array}{c}1.1244 \\
96\end{array}$ & $\begin{array}{c}3.30975 \\
8\end{array}$ \\
\hline v6o & 0 & $\begin{array}{c}0.445 \\
244\end{array}$ & $\begin{array}{c}0.3365 \\
34\end{array}$ & $\begin{array}{c}0.6624 \\
34\end{array}$ & $\begin{array}{c}1.56339 \\
6\end{array}$ \\
\hline v7i & 0 & $\begin{array}{c}1.658 \\
171\end{array}$ & $\begin{array}{c}0.5824 \\
52\end{array}$ & $\begin{array}{c}1.6836 \\
18\end{array}$ & $\begin{array}{c}5.16959 \\
1\end{array}$ \\
\hline $\mathrm{v} 7 \mathrm{o}$ & 0 & $\begin{array}{c}0.372 \\
525\end{array}$ & $\begin{array}{c}1.2758 \\
37\end{array}$ & $\begin{array}{c}4.5337 \\
89\end{array}$ & $\begin{array}{c}2.85513 \\
4\end{array}$ \\
\hline $\mathrm{v} 8 \mathrm{i}$ & $\begin{array}{l}3.5147 \\
9 \mathrm{E}-15\end{array}$ & $\begin{array}{c}1.147 \\
137\end{array}$ & $\begin{array}{c}1.6471 \\
22\end{array}$ & $\begin{array}{c}1.5797 \\
17\end{array}$ & $\begin{array}{c}1.11013 \\
1\end{array}$ \\
\hline v8o & 0 & $\begin{array}{c}2.002 \\
127\end{array}$ & $\begin{array}{c}1.9224 \\
83\end{array}$ & $\begin{array}{c}1.0004 \\
77\end{array}$ & $\begin{array}{c}1.28950 \\
8\end{array}$ \\
\hline
\end{tabular}

\subsection{Validation}

In standard method of evaluating segmented medical images registration is to use Sorenson's Dice coefficient [14] where a value of ' 1 ' indicates complete overlap in $2 \mathrm{~d}$ space and a ' 0 ' indicates no overlap. In order to evaluate the proposed method where the registration has been compared with linear overlaps, 2 of the 15 branches (branch 0 of both set 1 and set 4) were used to calculate their dice coefficient values according to the algorithm described [15] with a difference of $1 \mathrm{~mm}$. Later on, average, maximum and minimum values for both the sets dice indices have been calculated which are listed in table 2 . It can be seen from table 2 that -

- For set 2 the average dice value indicates a close to fully overlapped condition with deviations within a range of 0.1 where the maximum difference between quartile distributions from distance matrices falls within the range of 0.15 .

- $\quad$ For set 4 , the average value of dice is 0.7679 with deviations of about 0.2 while the difference in distribution of distances are as high as about an order of $3 \mathrm{~mm}$.

From these observations, it can be concluded that the proposed hierarchical registration and following distance matrix comparison can be considered as an alternative method for super imposing intra-patient blood vessels with temporal difference followed by vessel straightening comparison with their distance matrix distribution. 


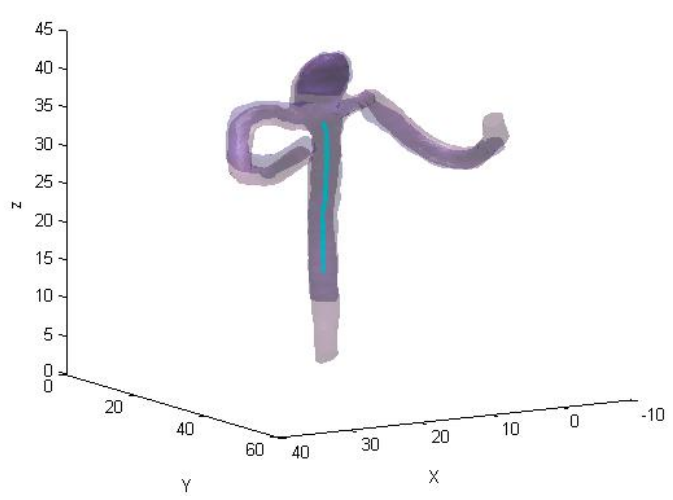

Fig 17: Dice index for a single branch (Set 4)

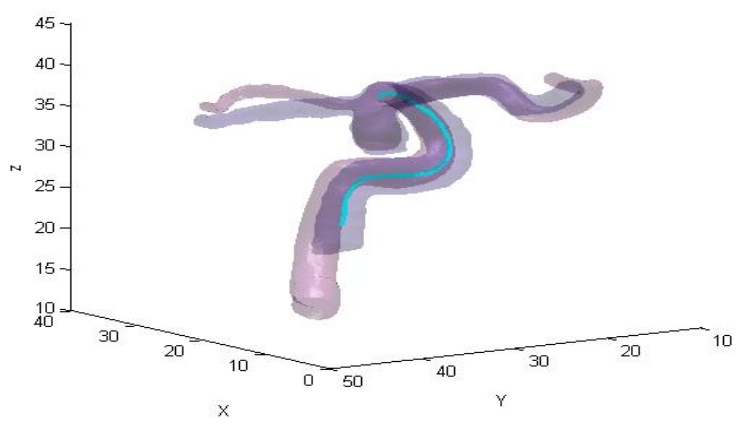

Fig 18: Dice index for a single branch (Set 4)

Table 2: Overlap measure with Dice index

\begin{tabular}{|l|l|l|l|}
\hline & average & $\min$ & $\max$ \\
\hline V2-1 & 0.8227 & 0.7212 & $\mathbf{0 . 9 2 4 1}$ \\
\hline V4-1 & 0.7679 & 0.5723 & $\mathbf{0 . 8 8 3 2}$ \\
\hline
\end{tabular}

\section{CONCLUSION}

This article proposes a hierarchical registration algorithm, especially for pre segmented cerebral blood vessels where there is a shortage of landmarks required for atlas based registration. The algorithm works in a semi-automated fashion where the landmark is identified from the bifurcating arterial zones. In order to verify the proposed method distance matrices were created to measure distortion in structural characteristics. Again, the results found from distribution matrices were verified with dice indices which indicated the proposed sequence can be used as an alternative measure of calculating comparative tortuosity between intra-patient vascular models with temporals differences.

\section{ACKNOWLEDGMENTS}

Our special thanks to $\mathrm{Mr}$. Al Imran for his valuable suggestions on some of the technical details for this study.

\section{REFERENCES}

[1] Ma, Z. et al., 2010. A Review of Algorithms for Medical Image Segmentation and their Applications to the Female Pelvic Cavity A Review of Algorithms for Medical Image Segmentation and their Applications to the Female Pelvic Cavity. Computer methods of biomechanical and biomedical engineering, 13(2), pp.235-246.

[2] Pham, D.L., Xu, C. \& Prince, J.L., 2000. A Survey of Current Methods in Medical Image Segmentation. Annual Review of Biomedical Engineering, 2, pp.315337.

[3] Afroz, A.S., An Alternative Approach of Evaluating Dice Similarity Index for Pre-segmented Blood Vessels. In Software, Knowledge, Information Management and Applications (SKIMA), 2014 8th International Conference on. IEEE, 2014. Dhaka, pp. 4-7.

[4] Hilsmann, A. et al., 2007. Deformable 4DCT lung registration with vessel bifurcations. In In International Conference of Computer Assisted Radiology and Surgery (CARS).

[5] Matsopoulos, G.K. et al., Multimodal registration of retinal images using self organizing maps. Medical Imaging, IEEE Transactions, 23(12), pp.1557-1563.

[6] Hsu, C.-Y. et al., 2015. Medical Image Processing for Fully Integrated Subject Specific Whole Brain Mesh Generation. Technologies, 3(2), pp.126-141. Available at: http://www.mdpi.com/2227-7080/3/2/126/ [Accessed October 13, 2015].

[7] Wang, F. \& Baba C. Vemuri., 2005. Simultaneous registration and segmentation of anatomical structures from brain MRI. Medical Image Computing and Computer-Assisted Intervention-MICCAI 2005., pp.1725.

[8] Prasad, G., 2013. Brain Mapping Methods: Segmentation, Registration, and Connectivity Analysis.

[9] Han, X. \& Fischl, B., 2007. Atlas renormalization for improved brain MR image segmentation across scanner platforms. Medical Imaging, IEEE Transactions, 26(4), pp.479-486.

[10] Khan, A.R., Chung, M.K. \& Beg, M.F., 2009. Robust atlas-based brain segmentation using multi-structure confidence-weighted registration. In Medical Image Computing and Computer-Assisted InterventionMICCAI 2009, pp.549-557.

[11] Antiga, L., 2002. Patient-specific modeling of geometry and blood flow in large arteries. Politecnico di Milano.

[12] De Bock, S. et al., 2012. Our capricious vessels: The influence of stent design and vessel geometry on the mechanics of intracranial aneurysm stent.

[13] Ma, D. et al., 2011. FINITE ELEMENT STUDY OF CONFORMITY OF FLOW DIVERTER WITH. In ASME 2011 Summer Bioengineering Conference SBC2011. pp. 1-2.

[14] Anon, Sørensen-Dice coefficient. Available at: http://en.wikipedia.org/wiki/Sorensen?Dice_coefficient [Accessed September 19, 2014]. 